\title{
Time- or Space-Dependent Coefficient Recovery in Parabolic Partial Differential Equation for Sensor Array in the Biological Computing
}

\author{
Guanglu Zhou, ${ }^{1}$ Boying Wu, ${ }^{2}$ Wen $\mathrm{Ji}^{3}{ }^{3}$ and Seungmin $\mathrm{Rho}^{4}$ \\ ${ }^{1}$ Department of Computer Science, Harbin Institute of Technology at Weihai, Weihai, Shandong 264209, China \\ ${ }^{2}$ Department of Mathematics, Harbin Institute of Technology, Harbin, Heilongjiang 150001, China \\ ${ }^{3}$ Beijing Key Laboratory of Mobile Computing \& Pervasive Device, Institute of Computing Technology, Beijing, China \\ ${ }^{4}$ Department of Multimedia, Sungkyul University, Anyō, Gyeonggi 100190, Republic of Korea
}

Correspondence should be addressed to Guanglu Zhou; guanglu_zhou_hit@163.com

Received 3 November 2014; Revised 18 December 2014; Accepted 19 December 2014

Academic Editor: Bo-Wei Chen

Copyright (C) 2015 Guanglu Zhou et al. This is an open access article distributed under the Creative Commons Attribution License, which permits unrestricted use, distribution, and reproduction in any medium, provided the original work is properly cited.

\begin{abstract}
This study presents numerical schemes for solving a parabolic partial differential equation with a time- or space-dependent coefficient subject to an extra measurement. Through the extra measurement, the inverse problem is transformed into an equivalent nonlinear equation which is much simpler to handle. By the variational iteration method, we obtain the exact solution and the unknown coefficients. The results of numerical experiments and stable experiments imply that the variational iteration method is very suitable to solve these inverse problems.
\end{abstract}

\section{Introduction}

Various inverse problems in a parabolic partial differential equation are widely encountered in modeling physical phenomena [1-3]. There are three kinds of inverse parameter problems of parabolic partial differential equations, including determining an unknown time-dependent coefficient, an unknown space-dependent coefficient, and an unknown source term.

The aim of this paper is to find $(u(x, t), a(x, t))$ in the parabolic equation

$$
\begin{gathered}
\frac{\partial u}{\partial t}=\frac{\partial}{\partial x}\left(a(x, t) \frac{\partial u}{\partial x}\right)+f(u, x, t), \\
0<x<L, \quad 0<t<T,
\end{gathered}
$$

where $a(x, t)$ is only a function with respect to $x$ or $t$.

When $a(x, t)=a(t)$, the boundary conditions and an extra measurement of (1) are as follows:

$$
\begin{array}{ll}
u(x, 0)=f(x), & 0 \leq x \leq 1, \\
u(0, t)=g_{0}(t), & 0 \leq t \leq T,
\end{array}
$$

$$
\begin{aligned}
u(1, t) & =g_{1}(t), \quad 0 \leq t \leq T, \\
u\left(x^{*}, t\right) & =E(t), \quad 0 \leq t \leq T, x^{*} \in(0,1),
\end{aligned}
$$

where $f(u, x, t)=\omega(t) u(x, t)+\phi(x, t), \phi(x, t), \omega(t), f(x)$, $g_{0}(t), g_{1}(t)$, and $E(t)$ are known functions. This equation is widely used to determine the unknown properties of a region by measuring only data on its boundary or a specified location in the domain. These unknown properties such as the conductivity medium are important to the physical process but usually cannot be measured directly or are very expensive to be measured. The existence and uniqueness of the solution to this problem are discussed in $[4,5]$.

There are various numerical methods to solve (1) and (2) or similar problems. Now we give a quick review of the previous work placed to our problem. Cannon [6] reduced the problem to a nonlinear integral equation for the coefficient $a(t)$. This approach works well for a parabolic equation in one space variable but does not easily extend to higher-dimensional problems because it depends on the 
explicit form of the fundamental solution of the heat operator. In Cannon and Yin [7], this problem was studied from a different point of view. The authors first transformed a large class of parabolic inverse problems into a nonclassical parabolic equation whose coefficients consist of trace type functional on the solution and its derivatives subject to some initial and boundary conditions. For the resulted nonclassical problem, they introduced a variation form by defining a new function; then both continuous and discrete Galerkin procedures are employed to the nonclassical problem. Authors of [8] presented the backward Euler finite difference scheme. It is shown that this scheme is stable in the maximum norm and error estimation was obtained. In [9], several firstand second-order finite difference numerical schemes have been developed to solve the nonclassical problem which is obtained by applying the transformation technique in [7] to problem (1) and (2). Also, a method is proposed in [10] to solve this problem which is based on a semianalytical approach. Authors of [11] used the pseudospectral Legendre method to solve this problem. An unconditionally stable efficient fourth-order numerical algorithm based on the functional transformation, the Pade approximation, and the Richardson extrapolation is proposed in [12] to compute the main function and the unknown time-dependent coefficient in (1). The Chebyshev cardinal functions are employed in [13] to recover the unknown coefficient. These schemes are efficient and easy to implement but the convergence order is low.

When $a(x, t)=a(x)$, the boundary conditions and an extra measurement of (1) are as follows:

$$
\begin{aligned}
& u(x, 0)=u_{0}(x), \quad 0 \leq x \leq L, \\
& \left.\frac{\partial u}{\partial x}\right|_{x=0}=0, \quad 0 \leq t \leq T, \\
& u(1, t)=g(t), \quad 0 \leq t \leq T, \\
& u(x, T)=u_{1}(x), \quad 0 \leq x \leq L,
\end{aligned}
$$

where $u_{0}(x), u_{1}(x), f(u, x, t)=f(x, t)$, and $g(t)$ are known functions. It is widely known that this model describes the heat conduction procedure in a given inhomogeneous medium with some input source $f(x, t)$ and the coefficient $a(x)$ represents a heat conduction property, namely, the heat capacity. There are various numerical methods to solve (1) and (3)-(6) or similar problems. Deng et al. [14] applied the gradient iteration algorithm for obtaining the approximate solutions. Kansa method is used by [15] to solve problem (1) and (2) and the stable experiments are given. Authors in [16] give an iterative fixed point projection method for this problem. In addition, there are other methods [17-22].

Although there are many methods for recovering the above inverse problems, those methods only give approximate solution. So it is worth noting that the variational iteration method can give the exact solution.

Professor $\mathrm{He}$ proposed variational iteration method (VIM) firstly in 1998 [23] and developed quickly VIM in 2006 and 2007. Based on the use of Lagrange multipliers for the identification of optimal values of parameters in a functional, VIM gives rapidly convergent successive approximations of the exact solution if such a solution exists. There are three standard variational iteration algorithms [24], called VIM-I, VIM-II, and VIM-III, for solving differential difference equations, integrodifferential equations, fractional differential equations, and fractal differential equations. These three forms of VIM have been proved by many authors to be a powerful mathematical tool for addressing various kinds of linear and nonlinear problems [25-28]. The reliability of the method and the reduction in the burden of computational work give this method wider application [29$32]$. In addition, some reviews can be found in $\mathrm{He}[24,33,34]$. Since the applications of VIM in inverse problems are very few, we use VIM-I to recover the unknown coefficients here. Furthermore, VIM gives the exact solution of this problem. Thus the variational iteration method is suitable for finding the approximation solution of the problem.

The rest of the paper is organized in four sections including Introduction. Section 2 gives the detailed progress and proof for recovering the unknown coefficients by applying VIM. In Section 3, numerical examples and a stable experiment are presented to imply the accuracy of VIM. Finally, a brief conclusion ends this paper.

\section{Application of He's Variational Iteration Method}

In this section, we will apply He's variational iteration method (VIM) to recover time- or space-dependent coefficient problems. The detailed introduction of VIM can be found in $[24,33,34]$.

2.1. Recovering Time-Dependent Coefficients. Using (1) and (2), we obtain

$$
\begin{aligned}
E^{\prime}(t) & =u_{t}\left(x^{*}, t\right) \\
& =a(t) u_{x x}\left(x^{*}, t\right)+\omega(t) E(t)+\phi\left(x^{*}, t\right) .
\end{aligned}
$$

Assuming that $u_{x x}\left(x^{*}, t\right) \neq 0$, we have

$$
a(t)=\frac{E^{\prime}(t)-\omega(t) E(t)-\phi\left(x^{*}, t\right)}{u_{x x}\left(x^{*}, t\right)} .
$$

Therefore the inverse problem (1) and (2) is equivalent to the following problem:

$$
\begin{aligned}
& u_{t}=\frac{E^{\prime}(t)-\omega(t) E(t)-\phi\left(x^{*}, t\right)}{u_{x x}\left(x^{*}, t\right)} u_{x x}+\omega(t) u \\
& +\phi(x, t), \quad 0<x<1,0<t<T, \\
& u(x, 0)=f(x), \quad 0 \leq x \leq 1, \\
& u(0, t)=g_{0}(t), \quad 0 \leq t \leq T, \\
& u(1, t)=g_{1}(t), \quad 0 \leq t \leq T .
\end{aligned}
$$


From (9),

$$
\begin{aligned}
-u_{t} & +\frac{E^{\prime}(t)-\omega(t) E(t)-\phi\left(x^{*}, t\right)}{u_{x x}\left(x^{*}, t\right)} u_{x x}+\omega(t) u \\
& +\phi(x, t)=0 .
\end{aligned}
$$
tion:

Constructing a correction function for the above equa-

$$
\begin{aligned}
u_{n+1}(x, t)= & u_{n}(x, t) \\
+\int_{x^{*}}^{x} \lambda(y)( & \\
& u_{n y y}(y, t)-\widetilde{u}_{n y y}(y, t)-\widetilde{u}_{n s}(y, t) \\
& +\frac{E^{\prime}(t)-\omega(t) E(t)-\phi\left(x^{*}, t\right)}{u_{n x x}\left(x^{*}, t\right)} \\
& \cdot \widetilde{u}_{n y y}(y, t) \\
& \left.+\omega(t) \widetilde{u}_{n}(y, t)+\phi(y, t)\right) d y .
\end{aligned}
$$

In the following, we determine the Lagrange multiplier $\lambda$ via variation theory:

$$
\begin{aligned}
& \delta u_{n+1}(x, t)=\delta u_{n}(x, t) \\
&+\delta \int_{x^{*}}^{x} \lambda(y)\left(u_{n y y}(y, t)-\widetilde{u}_{n y y}(y, t)-\widetilde{u}_{n s}(y, t)\right. \\
&+\frac{E^{\prime}(t)-\omega(t) E(t)-\phi\left(x^{*}, t\right)}{u_{n x x}\left(x^{*}, t\right)} \\
& \cdot \widetilde{u}_{n y y}(y, t) \\
&\left.+\omega(t) \widetilde{u}_{n}(y, t)+\phi(y, t)\right) d y .
\end{aligned}
$$

$$
\text { Applying } \delta \tilde{u}_{n}=0 \text {, then }
$$

$$
\begin{aligned}
\delta u_{n+1}(x, t)= & \delta u_{n}(x, t)\left(1-\lambda^{\prime}(x)\right)+\delta u_{n}^{\prime}(x, t) \lambda(x) \\
& -\int_{x^{*}}^{x} \lambda^{\prime \prime}(y) \delta u_{n}(y, t) d y=0,
\end{aligned}
$$

so

$$
\begin{aligned}
\lambda^{\prime \prime}(y) & =0, \\
1-\lambda^{\prime}(x) & =0, \\
\lambda(x) & =0 .
\end{aligned}
$$

Thus $\lambda(y)=y-x$; this gives the iterative formula:

$$
\begin{aligned}
u_{n+1}(x, t)= & u_{n}(x, t) \\
+\int_{x^{*}}^{x}(y-x)( & -u_{n s}(y, t) \\
& +\frac{E^{\prime}(t)-\omega(t) E(t)-\phi\left(x^{*}, t\right)}{u_{n x x}\left(x^{*}, t\right)} \\
& \cdot u_{n y y}(y, t) \\
& \left.+\omega(t) u_{n}(y, t)+\phi(y, t)\right) d y .
\end{aligned}
$$

Now, take $u_{0}(x, t)$ and $\partial_{x x} u_{0}\left(x^{*}, t\right) \rightarrow \partial_{x x} u\left(x^{*}, t\right)$ as an initial value. By (18), we can obtain the $n$-order approximate solution $u_{n}(x, t)$ of (9). Putting

$$
h_{n}(t)=\frac{E^{\prime}(t)-\omega(t) E(t)-\phi\left(x^{*}, t\right)}{u_{n x x}\left(x^{*}, t\right)},
$$

then

$$
\begin{aligned}
& u_{n+1}(x, t)= u_{n}(x, t) \\
&+\int_{x^{*}}^{x}(y-x)\left(-u_{n s}(y, t)+h_{n}(t) u_{n y y}(y, t)\right. \\
&\left.\quad+\omega(t) u_{n}(y, t)+\phi(y, t)\right) d y
\end{aligned}
$$

and its derivative about $x$ :

$$
\begin{aligned}
\partial_{x} u_{n+1}(x, t)= & \partial_{x} u_{n}(x, t) \\
-\int_{x^{*}}^{x} & \left(-u_{n s}(y, t)+h_{n}(t) u_{n y y}(y, t)\right. \\
& \left.+\omega(t) u_{n}(y, t)+\phi(y, t)\right) d y
\end{aligned}
$$

and the derivative of the above about $x$ :

$$
\begin{aligned}
\partial_{x x} u_{n+1}(x, t)= & \partial_{x x} u_{n}(x, t) \\
& -\left(-u_{n t}(x, t)+h_{n}(t) u_{n x x}(x, t)\right. \\
& \left.+\omega(t) u_{n}(x, t)+\phi(x, t)\right) .
\end{aligned}
$$

Inserting $x=x^{*}$, we obtain

$$
\begin{aligned}
\partial_{x x} u_{n+1}\left(x^{*}, t\right)= & \partial_{x x} u_{n}\left(x^{*}, t\right) \\
& -\left(-u_{n t}\left(x^{*}, t\right)+h_{n}(t) u_{n x x}\left(x^{*}, t\right)\right. \\
& \left.+\omega(t) u_{n}\left(x^{*}, t\right)+\phi\left(x^{*}, t\right)\right) .
\end{aligned}
$$

From (18), one can infer that

$$
\begin{aligned}
u_{n}\left(x^{*}, t\right) & =u_{n-1}\left(x^{*}, t\right)=\cdots=u_{0}\left(x^{*}, t\right) \\
& =E(t) u_{n t}\left(x^{*}, t\right)=u_{n-1 t}\left(x^{*}, t\right)=\cdots \\
& =u_{0 t}\left(x^{*}, t\right)=E^{\prime}(t),
\end{aligned}
$$


so

$$
\begin{aligned}
- & u_{n t}\left(x^{*}, t\right)+h_{n}(t) u_{n x x}\left(x^{*}, t\right) \\
& +\omega(t) u_{n}\left(x^{*}, t\right)+\phi\left(x^{*}, t\right) \\
= & -E^{\prime}(t)+\frac{E^{\prime}(t)-\omega(t) E(t)-\phi\left(x^{*}, t\right)}{u_{n x x}\left(x^{*}, t\right)} u_{n x x}\left(x^{*}, t\right) \\
& +\omega(t) E(t)+\phi\left(x^{*}, t\right) \\
= & 0
\end{aligned}
$$

which leads to the following:

$$
\partial_{x x} u_{n+1}\left(x^{*}, t\right)=\partial_{x x} u_{n}\left(x^{*}, t\right),
$$

so as to deduce

$$
\begin{aligned}
\partial_{x x} u_{n}\left(x^{*}, t\right) & =\partial_{x x} u_{n-1}\left(x^{*}, t\right)=\cdots=\partial_{x x} u_{0}\left(x^{*}, t\right) \\
& \longrightarrow \partial_{x x} u\left(x^{*}, t\right) .
\end{aligned}
$$

Therefore, by (8), the approximate solution $a_{n}(t)$ to $a(t)$ can be expressed in the following form:

$$
a_{n}(t)=\frac{E^{\prime}(t)-\omega(t) E(t)-\phi\left(x^{*}, t\right)}{u_{n x x}\left(x^{*}, t\right)} .
$$

2.2. Recovering Space-Dependent Coefficients. Using (1) and (3)-(6), we obtain

$$
\frac{\partial u}{\partial t}=\frac{\partial}{\partial x}\left(a(x) \frac{\partial u}{\partial x}\right)+f(x, t)
$$

then

$$
u_{t}(x, t)-f(x, t)=\frac{\partial}{\partial x}\left(a(x) \frac{\partial u}{\partial x}\right)
$$

putting $t=T$ and integrating the above equation with $x$ from 0 to $x$,

$$
\begin{aligned}
& \int_{0}^{x} u_{t}(w, T)-f(w, T) d w \\
& \quad=a(x)\left(u_{x}(x, T)-u_{x}(0, T)\right) ;
\end{aligned}
$$

applying condition (4),

$$
u_{x}(0, T)=0
$$

thus

$$
\int_{0}^{x} u_{t}(w, T)-f(w, T) d w=a(x) u_{x}(x, T) .
$$

Assuming that $u_{x}(x, T)=u_{1}^{\prime}(x) \neq 0$, we have

$$
a(x)=\frac{\int_{0}^{x} u_{t}(w, T)-f(w, T) d w}{u_{1}^{\prime}(x)} .
$$

Therefore, the inverse problem (1) and (3)-(6) is equivalent to the following problem:

$$
\begin{array}{r}
\frac{\partial u}{\partial t}=\frac{\partial}{\partial x}\left(\frac{\int_{0}^{x} u_{t}(w, T)-f(w, T) d w}{u_{1}^{\prime}(x)} \frac{\partial u}{\partial x}\right)+f(x, t), \\
0<x<L, \quad 0<t<T,
\end{array}
$$

with the initial condition

$$
u(x, 0)=u_{0}(x), \quad 0 \leq x \leq L
$$

and boundary conditions

$$
\begin{aligned}
\left.\frac{\partial u(x, t)}{\partial x}\right|_{x=0} & =0, \quad 0 \leq t \leq T, \\
u(1, t) & =g(t), \quad 0 \leq t \leq T .
\end{aligned}
$$

Next, we are concerned with the approximate solutions of (35)-(37) by the variational iteration method. Applying the variation theory, we can construct an iteration formula.

From (35),

$$
\begin{gathered}
\frac{\partial u}{\partial t}-\frac{\partial}{\partial x}\left(\frac{\int_{0}^{x} u_{t}(w, T)-f(w, T) d w}{u_{1}^{\prime}(x)} \frac{\partial u}{\partial x}\right) \\
-f(x, t)=0 .
\end{gathered}
$$

Constructing a correction function for the above equation,

$$
\begin{aligned}
& u_{n+1}(x, t) \\
& =u_{n}(x, t) \\
& +\int_{T}^{t} \lambda(s)\left(u_{n s}(x, s)-\partial_{x}\right. \\
& \cdot\left(\frac{\int_{0}^{x} \widetilde{u}_{n t}(w, T)-\tilde{f}(w, T) d w}{u_{1}^{\prime}(x)} \widetilde{u}_{n x}(x, s)\right) \\
& -\tilde{f}(x, s)) d s .
\end{aligned}
$$


In the following, we determine the Lagrange multiplier $\lambda$ via variation theory:

$$
\begin{aligned}
& \delta u_{n+1}(x, t) \\
& =\delta u_{n}(x, t) \\
& +\delta \int_{T}^{t} \lambda(s)\left(u_{n s}(x, s)-\partial_{x}\right. \\
& \\
& \qquad\left(\frac{\int_{0}^{x} \widetilde{u}_{n t}(w, T)-\tilde{f}(w, T) d w}{u_{1}^{\prime}(x)} \widetilde{u}_{n x}(x, s)\right) \\
& -\tilde{f}(x, s)) d s .
\end{aligned}
$$

Applying $\delta \tilde{u}_{n}=0$, then

$$
\begin{aligned}
\delta u_{n+1}(x, t)= & \delta u_{n}(x, t)(1+\lambda(t)) \\
& -\int_{T}^{t} \lambda^{\prime}(s) \delta u_{n}(x, s) d s=0,
\end{aligned}
$$

so

$$
\begin{aligned}
1+\lambda(t) & =0, \\
\lambda^{\prime}(s) & =0 .
\end{aligned}
$$

Thus $\lambda(s)=-1$; this gives the iterative formula

$$
\begin{aligned}
& u_{n+1}(x, t) \\
& =u_{n}(x, t) \\
& -\int_{T}^{t}\left(u_{n s}(x, s)-\partial_{x}\right. \\
& \cdot\left(\frac{\int_{0}^{x} u_{n t}(w, T)-f(w, T) d w}{u_{1}^{\prime}(x)} u_{n x}(x, s)\right) \\
& -f(x, s)) d s .
\end{aligned}
$$

Now, take $u_{0}(x, t)$ and $\partial_{x} u_{0}(x, T) \rightarrow u_{1}^{\prime}(x)$ as an initial value. By (43), we can obtain the $n$-order approximate solution $u_{n}(x, t)$ of $(35)$.

If $u_{n t}(x, T) \rightarrow u_{t}(x, T)$, then we can approximate to $a(x)$ by the following:

$$
a_{n}(x)=\frac{\int_{0}^{x} u_{n t}(w, T)-f(w, T) d w}{u_{1}^{\prime}(x)} .
$$

Now, we prove that $u_{n t}(x, T) \rightarrow u_{t}(x, T)$.

By (43), $\partial_{x} u_{n+1}(x, T)=\partial_{x} u_{n}(x, T)$, which leads to the following:

$$
\partial_{x} u_{n}(x, T)=\partial_{x} u_{n-1}(x, T)=\cdots=\partial_{x} u_{0}(x, T),
$$

so as to deduce

$$
u_{n t}(x, T) \longrightarrow u_{t}(x, T) \text {. }
$$

\section{Numerical Examples}

Example 1. Considering a special case of (1) and (2) with [9, 13],

$$
\begin{aligned}
\omega(t) & =0, \\
\phi(x, t) & =0, \\
f(x) & =e^{x / 2}, \\
g_{0}(t) & =\frac{1+2 t^{3}}{1+t^{3}}+\sin \left(\frac{t}{2}\right), \\
g_{1}(t) & =\frac{\sqrt{e}\left(1+2 t^{3}\right)}{1+t^{3}}+\sqrt{e} \sin \left(\frac{t}{2}\right), \\
E(t) & =\frac{1.13315\left(1+2 t^{3}\right)}{1+t^{3}}+1.13315 \sin \left(\frac{t}{2}\right),
\end{aligned}
$$

with $x^{*}=0.25$, for which the exact solution is

$$
\begin{aligned}
u(x, t) & =\frac{e^{x / 2}\left(1+2 t^{3}\right)}{1+t^{3}}+e^{x / 2} \sin \left(\frac{t}{2}\right) \\
a(t) & =\frac{2\left[6 t^{2}+\left(1+t^{3}\right)^{2} \cos (t / 2)\right]}{(1+t)^{3}\left[1+2 t^{3}+\left(1+t^{3}\right) \sin (t / 2)\right]}
\end{aligned}
$$

Beginning with

$$
u_{0}(x, t)=f(x)\left(a g_{0}(t)+b g_{1}(t)\right),
$$

where $a, b$ are the unknown parameters to be further determined, according to (18), one can obtain the first-order approximation $u_{1}(x, t)$, and we find

$$
u_{i}(x, t)=u_{1}(x, t), \quad i=2,3, \ldots
$$

Incorporating the initial condition $u_{0}(x, 0)=f(x)$, $u_{0}(0, t)=g_{0}(t), u_{0}(1, t)=g_{1}(t)$ of Example 1 into $u_{1}(x, t)$, the unknown parameters $a, b$ can be obtained. Therefore, the first-order approximation

$$
u_{1}(x, t)=\frac{e^{x / 2}\left(1+2 t^{3}\right)}{1+t^{3}}+e^{x / 2} \sin \left(\frac{t}{2}\right)
$$

is obtained, which is the exact solution of Example 1. From (28), we have

$$
a_{1}(t)=\frac{2\left[6 t^{2}+\left(1+t^{3}\right)^{2} \cos (t / 2)\right]}{(1+t)^{3}\left[1+2 t^{3}+\left(1+t^{3}\right) \sin (t / 2)\right]},
$$

which is equal to the exact $a(t)$ of Example 1 .

Example 2. Finding $a(t)$ in (1) and (2) with $[9,13]$,

$$
\omega(t)=0,
$$

$$
\phi(x, t)=(3+\cos (t)) e^{t} \cos (x),
$$




$$
\begin{aligned}
& f(x)=\cos (x), \\
& g_{0}(t)=e^{t}, \\
& g_{1}(t)=\cos (1) e^{t}, \\
& E(t)=\left(1+t+\frac{t^{2}}{2}\right) e^{-t},
\end{aligned}
$$

where $x^{*}=4 / 9$. The true solution is $u(x, t)=e^{t} \cos (x)$ while $a(t)=2+\cos (t)$.

Beginning with

$$
u_{0}(x, t)=f(x)\left(a g_{0}(t)+b g_{1}(t)\right)
$$

where $a, b$ are the unknown parameters to be further determined, according to (18), one can obtain the first-order approximation $u_{1}(x, t)$, and we find

$$
u_{i}(x, t)=u_{1}(x, t), \quad i=2,3, \ldots
$$

Incorporating the initial condition $u_{0}(x, 0)=f(x)$, $u_{0}(0, t)=g_{0}(t), u_{0}(1, t)=g_{1}(t)$ of Example 2 into $u_{1}(x, t)$, the unknown parameters $a, b$ can be obtained. Therefore, the first-order approximation $u_{1}(x, t)$ is obtained and $u_{1}(x, t)=$ $e^{t} \cos (x)$, which is the exact solution of Example 2. From (28), we have $a_{1}(t)=2+\cos (t)$, which is equal to the exact $a(t)$ of Example 2.

Example 3. We solve the problem (1) and (2) with $[9,13]$ :

$$
\begin{aligned}
\omega(t) & =-t^{2}-1, \\
\phi(x, t) & =2 t(x+t) e^{\left(t^{2}+1\right) x}, \\
f(x) & =e^{x} \\
g_{0}(t) & =1 \\
g_{1}(t) & =e^{t^{2}+1} \\
E(t) & =e^{2\left(t^{2}+1\right) / 7}
\end{aligned}
$$

whose true solution is $u(x, t)=e^{x\left(t^{2}+1\right)}$ while $a(t)=1 /(1+$ $\left.t^{2}\right)^{2}, x^{*}=2 / 7$.

Beginning with

$$
u_{0}(x, t)=f(x)\left(a_{0}(t)+b g_{1}(t)\right)
$$

where $a, b$ are the unknown parameters to be further determined, according to (18), one can obtain the first-order approximation $u_{1}(x, t)$, and we find

$$
u_{i}(x, t)=u_{1}(x, t), \quad i=2,3, \ldots
$$

Incorporating the initial condition $u_{0}(x, 0)=f(x)$, $u_{0}(0, t)=g_{0}(t), u_{0}(1, t)=g_{1}(t)$ of Example 3 into $u_{1}(x, t)$, the unknown parameters $a, b$ can be obtained. Therefore, the first-order approximation $u_{1}(x, t)$ is obtained and $u_{1}(x, t)=$ $e^{x\left(t^{2}+1\right)}$, which is the exact solution of Example 3. From (28), we have $a_{1}(t)=1 /\left(1+t^{2}\right)^{2}$, which is equal to the exact $a(t)$ of Example 3 .

The above three examples are about time-dependent coefficient; in the following we take space-dependent coefficient examples.

Applying the above VIM, we begin with $u_{0}(x, t)=$ $u_{0}(x)(a g(t)+b)$, where $a, b$ are the unknown parameters to be further determined. Incorporating the initial and boundary condition (36) and (37) into $u_{0}(x, t)$, the unknown parameters $a, b$ can be obtained. According to (43), one can obtain the first-order approximation $u_{1}(x, t)$. Here, $T=L=$ 1.

Example 4. We take the boundary conditions, initial condition, and additional specification function (3)-(6) as $[14,15]$

$$
\begin{gathered}
g(t)=e^{t}, \\
u_{0}(x)=x^{3}, \\
u_{1}(x)=e^{T} x^{3}, \\
f(x, t)=0,
\end{gathered}
$$

with the exact solution as

$$
u(x, t)=e^{t} x^{3}
$$

and the identifying coefficient as

$$
a(x)=\frac{1}{12} x^{2}
$$

Therefore, the first-order approximation $u_{1}(x, t)=(a+$ $\left.b+a^{2}\left(-1+e^{t}\right)+a b t\right) x^{3}$ is obtained. We can determine $a=$ $1, b=0$, so $u_{1}(x, t)=e^{t} x^{3}$ which is the exact solution of Example 4. From (44), we have $a_{1}(x)=(1 / 12) x^{2}$ which is equal to the exact $a(x)$ of Example 4 .

Example 5. Finding $a(x)$ in (1) and (3)-(6) in [15]

$$
\begin{gathered}
g(t)=e^{t+1}, \\
u_{0}(x)=x^{2} e^{x}, \\
u_{1}(x)=e^{x+T} x^{2}, \\
f(x, t)=0 .
\end{gathered}
$$

The true solution is $u(x, t)=e^{x+t} x^{2}$ while $a(x)=\left(e^{x}\left(x^{2}-\right.\right.$ $2 x+2)-2) / x(x+2) e^{x}$.

From (43), the first-order approximate solution

$$
\begin{aligned}
& u_{1}(x, t) \\
& \quad=e^{x}\left(-a e^{2}\left(-1+a\left(e-e^{t}\right)\right)+b(1+a e(-1+t))\right) x^{2} .
\end{aligned}
$$

Incorporating the initial conditions, we determine $a=1 / e$, $b=0$. Therefore, the first-order approximation $u_{1}(x, t)$ is 


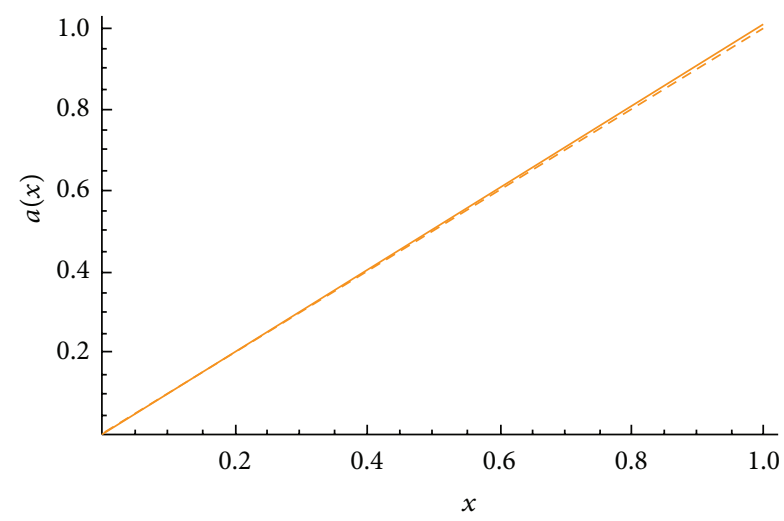

FIgURE 1: Numerical solutions $a(x)$ of Example 6 with $\operatorname{random}(x) \epsilon$ $[-1,1]$.

obtained and $u_{1}(x, t)=e^{x+t} x^{2}$ which is the exact solution of Example 5. From (44), we have $a_{1}(x)=\left(e^{x}\left(x^{2}-2 x+2\right)-\right.$ $2) / x(x+2) e^{x}$ which is equal to the exact $a(x)$ of Example 5 .

Example 6. We solve the problem (1) and (3)-(6) in [15]:

$$
\begin{aligned}
g(t) & =e^{t}, \\
u_{0}(x) & =x^{3}, \\
u_{1}(x) & =e^{T} x^{3}, \\
f(x, t) & =x^{3} e^{t}-9 x^{2} e^{t},
\end{aligned}
$$

whose true solution is $u(x, t)=x^{3} e^{t}$ while $a(x)=x$.

We can determine $a=1, b=0$. Therefore, the first-order approximation $u_{1}(x, t)=x^{2}\left(\left(b+a e^{t}\right) x+\left((-1+a)\left(-e+e^{t}\right)+\right.\right.$ $b(-1+t))(9+(-1+a) x))=e^{t} x^{3}$, which is the exact solution of Example 6. From (44), we have $a_{1}(x)=x$ which is equal to the exact $a(x)$ of Example 6.

In order to imply the stability of this method, we perturb the additional specification data $u_{1}(x)$ as

$$
u_{1}^{\delta}(x)=u_{1}(x)[1+\delta \times \operatorname{random}(x)]
$$

with $\delta=1 \%$; the reconstruction results are also stable, see Figure 1.

\section{Conclusion}

The VIM has been applied in solving a variety of equations, but it was rarely applied in inverse problems. Here, we develop the new application area of VIM; our contribution is that we apply VIM to solve the inverse problem of time- or spacedependent coefficients in a parabolic partial differential equation and obtain the exact solution. The numerical results fully demonstrate the superiority of VIM for these inverse problems.

\section{Appendix}

To imagine the basic idea behind He's VIM, we consider the following general differential equation:

$$
L u(x, t)+R u(x, t)+N u(x, t)=g(x, t),
$$

where $L$ is the highest order derivative that is assumed to be easily invertible, $R$ is a linear differential operator of order less than $L, N u$ represents the nonlinear terms, and $g$ is a source term. The basic characteristic of He's method is to construct a correction function for (A.1), which reads

$$
\begin{aligned}
& u_{n+1}(x, t) \\
& =u_{n}(x, t)+\int_{0}^{x} \lambda(y)\left(L u_{n}(y, t)+R \widetilde{u}_{n}(y, t)\right. \\
& \left.+N \widetilde{u}_{n}(y, t)-g(y, t)\right) d y,
\end{aligned}
$$

where $\lambda$ is a Lagrange multiplier which can be identified optimally via variation theory, $u_{n}$ is the $n$th approximate solution, and $\widetilde{u}_{n}$ denotes a restricted variation; that is, $\delta \widetilde{u}_{n}=$ 0 .

To solve (A.1) by He's VIM, we first determine the Lagrange multiplier $\lambda$ that can be identified optimally via variation theory. Then, the successive approximations $u_{n}(x, t), n=0,1,2, \ldots$, of the solution $u(x, t)$ can be readily obtained upon using the obtained Lagrange multiplier and any selective function $u_{0}$. Consequently, the exact solution may be obtained by using

$$
u(x, t)=\lim _{n \rightarrow+\infty} u_{n}(x, t) .
$$

In summary, we have the following variation iteration formula:

$$
\begin{aligned}
u_{n+1}(x, t)= & u_{n}(x, t) \\
& +\int_{0}^{x} \lambda(y)\left(L u_{n}(y, t)+R u_{n}(y, t)\right. \\
& \left.+N u_{n}(y, t)-g(y, t)\right) d y,
\end{aligned}
$$

where $u_{0}(x, t)$ is an arbitrary function satisfying initial and boundary conditions.

It should be specially pointed out that the more accurate the identification of the multiplier is, the faster the approximations converge to their exact solutions.

Remark 7. We cite an integrate of $x$ in (A.2) as an example; one needs an integrate of $t$ by a similar method.

\section{Conflict of Interests}

The authors declare that there is no conflict of interests regarding the publication of this paper.

\section{Acknowledgment}

The authors would like to thank the unknown referees for their careful reading and helpful comments. 


\section{References}

[1] M. Dehghan, "An inverse problem of finding a source parameter in a semilinear parabolic equation," Applied Mathematical Modelling, vol. 25, no. 9, pp. 743-754, 2001.

[2] M. Dehghan, "Identification of a time-dependent coefficient in a partial differential equation subject to an extra measurement," Numerical Methods for Partial Differential Equations, vol. 21, no. 3, pp. 611-622, 2005.

[3] M. Dehghan, "Parameter determination in a partial differential equation from the overspecified data," Mathematical and Computer Modelling, vol. 41, no. 2-3, pp. 197-213, 2005.

[4] J. R. Cannon and W. Rundell, "Recovering a time dependent coefficient in a parabolic differential equation," Journal of Mathematical Analysis and Applications, vol. 160, no. 2, pp. 572582, 1991.

[5] A. I. Prilepko, D. G. Orlovsky, and I. A. Vasin, Methods for Solving Inverse Problems in Mathematical Physics, vol. 1, Marcel Dekker, New York, NY, USA, 2000.

[6] J. R. Cannon, "Determination of an unknown coefficient in a parabolic differential equation," Duke Mathematical Journal, vol. 30, pp. 313-323, 1963.

[7] J. R. Cannon and H. M. Yin, "Numerical solutions of some parabolic inverse problems," Numerical Methods for Partial Differential Equations, vol. 2, pp. 177-191, 1990.

[8] H. Azari, W. Allegretto, Y. Lin, and S. Zhang, "Numerical procedures for recovering a time dependent coefficient in a parabolic differential equation," Dynamics of Continuous, Discrete and Impulsive Systems Series B: Applications and Algorithms, vol. 11, no. 1-2, pp. 181-199, 2004.

[9] M. Dehghan, "Identification of a time-dependent coefficient in a partial differential equation subject to an extra measurement," Numerical Methods for Partial Differential Equations, vol. 21, no. 3, pp. 611-622, 2005.

[10] M. Dehghan and M. Tatari, "Solution of a parabolic equation with a time-dependent coefficient and an extra measurement using the decomposition procedure of adomian," Physica Scripta, vol. 72, no. 6, pp. 425-431, 2005.

[11] M. Shamsi and M. Dehghan, "Recovering a time-dependent coefficient in a parabolic equation from overspecified boundary data using the pseudospectral legendre method," Numerical Methods for Partial Differential Equations, vol. 23, no. 1, pp. 196210, 2007.

[12] W. Liao, M. Dehghan, and A. Mohebbi, "Direct numerical method for an inverse problem of a parabolic partial differential equation," Journal of Computational and Applied Mathematics, vol. 232, no. 2, pp. 351-360, 2009.

[13] M. Lakestani and M. Dehghan, "The use of Chebyshev cardinal functions for the solution of a partial differential equation with an unknown time-dependent coefficient subject to an extra measurement," Journal of Computational and Applied Mathematics, vol. 235, no. 3, pp. 669-678, 2010.

[14] Z.-C. Deng, L. Yang, J.-N. Yu, and G.-W. Luo, "Identifying the diffusion coefficient by optimization from the final observation," Applied Mathematics and Computation, vol. 219, no. 9, pp. 4410-4422, 2013.

[15] K. Parand and J. A. Rad, "Kansa method for the solution of a parabolic equation with an unknown spacewise-dependent coefficient subject to an extra measurement," Computer Physics Communications, vol. 184, no. 3, pp. 582-595, 2013.

[16] A. Golayoglu Fatullayev and S. Cula, "An iterative procedure for determining an unknown spacewise-dependent coefficient in a parabolic equation," Applied Mathematics Letters, vol. 22, no. 7, pp. 1033-1037, 2009.

[17] F. Hettlich and W. Rundell, "Identification of a discontinuous source in the heat equation," Inverse Problems, vol. 17, no. 5, pp. 1465-1482, 2001.

[18] Z. Yi and D. A. Murio, "Source term identification in 1-D IHCP," Computers and Mathematics with Applications, vol. 47, no. 12, pp. 1921-1933, 2004.

[19] A. Hasanov, "Simultaneous determination of source terms in a linear parabolic problem from the final overdetermination: weak solution approach," Journal of Mathematical Analysis and Applications, vol. 330, no. 2, pp. 766-779, 2007.

[20] L. Yang, Z. C. Deng, J. N. Yu, and G. W. Luo, "Two regularization strategies for an evolutional type inverse heat source problem," Journal of Physics A: Mathematical and Theoretical, vol. 42, Article ID 365203, p. 16, 2009.

[21] L. Yang, Z.-C. Deng, J.-N. Yu, and G.-W. Luo, "Optimization method for the inverse problem of reconstructing the source term in a parabolic equation," Mathematics and Computers in Simulation, vol. 80, no. 2, pp. 314-326, 2009.

[22] J. Peralta and L. E. Olivar, "Regularization algorithm within two parameters for the identification of the heat conduction coefficient in the parabolic equation," Mathematical and Computer Modelling, vol. 57, no. 7-8, pp. 1990-1998, 2013.

[23] J.-H. He, "Approximate analytical solution for seepage flow with fractional derivatives in porous media," Computer Methods in Applied Mechanics and Engineering, vol. 167, no. 1-2, pp. 57-68, 1998.

[24] J.-H. He, "Variational iteration method-some recent results and new interpretations," Journal of Computational and Applied Mathematics, vol. 207, no. 1, pp. 3-17, 2007.

[25] Y. Aksoy, M. Pakdemirli, S. Abbasbandy, and H. Boyaci, "New perturbation-iteration solutions for nonlinear heat transfer equations," International Journal of Numerical Methods for Heat and Fluid Flow, vol. 22, no. 7, pp. 814-828, 2012.

[26] A. Yildirim and H. Koçak, "Rational approximation solution of the foam drainage equation with time- and space-fractional derivatives," International Journal of Numerical Methods for Heat and Fluid Flow, vol. 22, no. 4, pp. 512-525, 2012.

[27] S. M. Mehdi Hosseini, S. T. Mohyud-Din, and H. Ghaneai, "Variational iteration method for Hirota-Satsuma coupled KDV equation using auxiliary Parameter," International Journal of Numerical Methods for Heat and Fluid Flow, vol. 22, no. 3, pp. 277-286, 2012.

[28] H. Ghaneai, M. M. Hosseini, and S. T. Mohyud-Din, "Modified variational iteration method for solving a neutral functionaldifferential equation with proportional delays," International Journal of Numerical Methods for Heat and Fluid Flow, vol. 22, no. 8, pp. 1086-1095, 2012.

[29] F. Geng and Y. Lin, "Application of the variational iteration method to inverse heat source problems," Computers and Mathematics with Applications, vol. 58, no. 11-12, pp. 2098-2102, 2009.

[30] D. D. Ganji and A. Sadighi, "Application of homotopyperturbation and variational iteration methods to nonlinear heat transfer and porous media equations," Journal of Computational and Applied Mathematics, vol. 207, no. 1, pp. 24-34, 2007.

[31] M. Dehghan, M. Tatari, and A. Azizi, "The solution of the Falkner-Skan equation arising in the modelling of boundarylayer problems via variational iteration method," International Journal of Numerical Methods for Heat and Fluid Flow, vol. 21, no. 2, pp. 136-149, 2011. 
[32] G. E. Drăgănescu and V. Căpălnăşăn, "Nonlinear relaxation phenomena in polycrystalline solids," International Journal of Nonlinear Sciences and Numerical Simulation, vol. 4, no. 3, pp. 219-225, 2003.

[33] J.-H. He, "A short remark on fractional variational iteration method," Physics Letters. A, vol. 375, no. 38, pp. 3362-3364, 2011.

[34] J.-H. He, "Asymptotic methods for solitary solutions and compactons," Abstract and Applied Analysis, vol. 2012, Article ID 916793, 130 pages, 2012. 


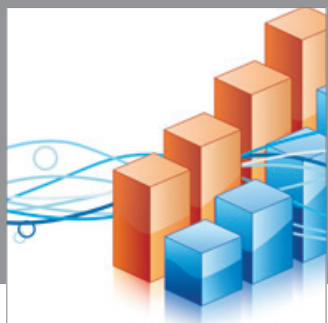

Advances in

Operations Research

mansans

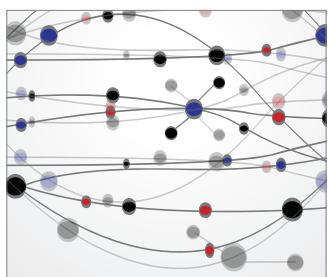

The Scientific World Journal
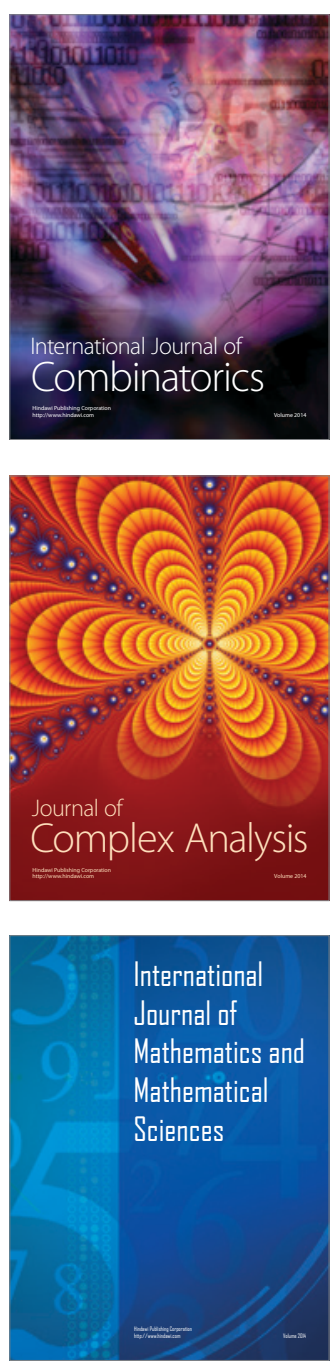
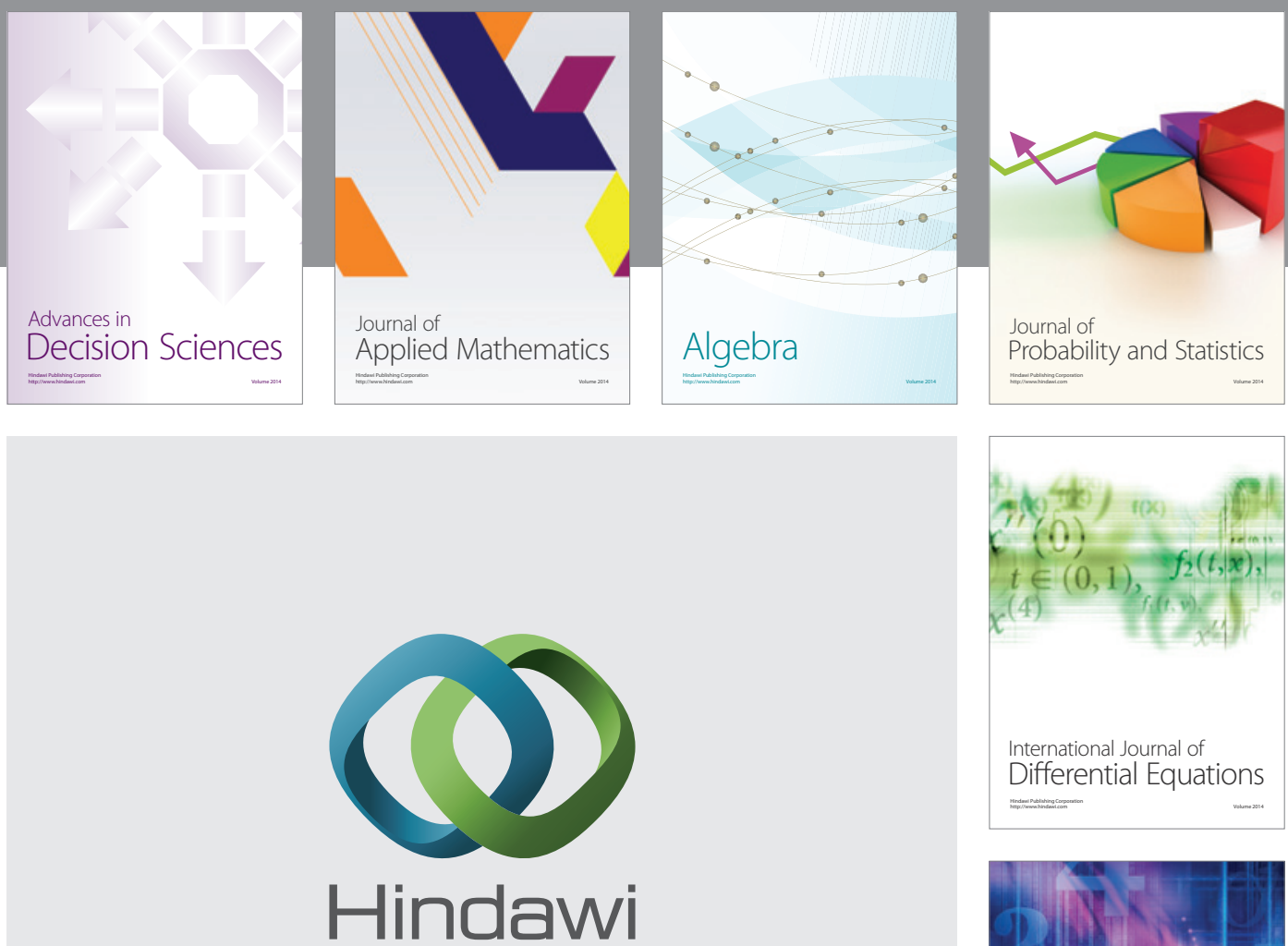

Submit your manuscripts at http://www.hindawi.com
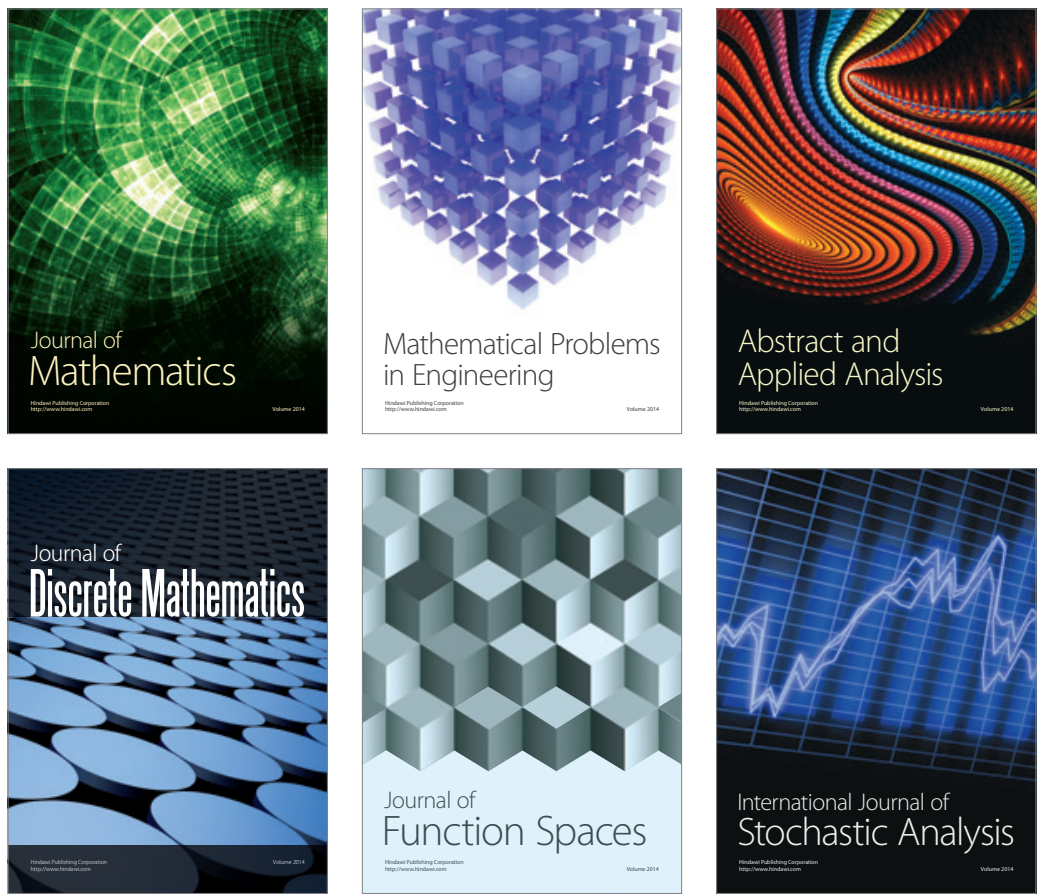

Journal of

Function Spaces

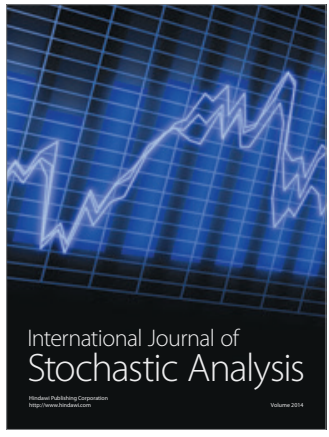

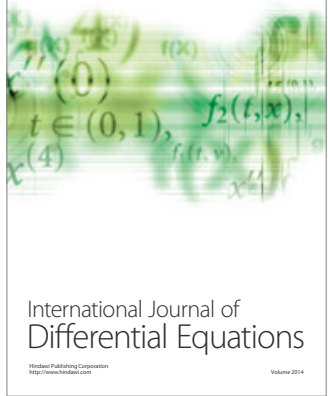
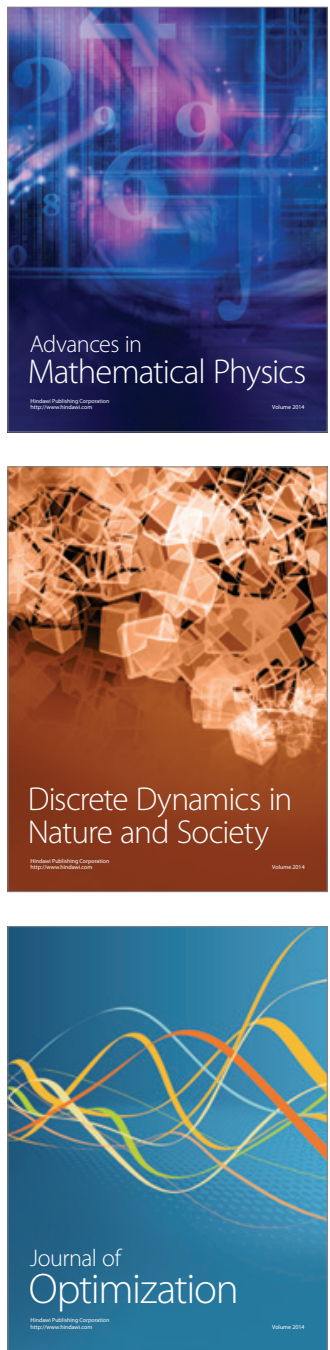